\section{Vorschlag für ein neues System progressiver Umweltabgaben}

In seiner jüngst erschienen Dissertation: Neue ökonomische Instrumente in der staatlichen Umweltpolitik (Frankfurt/M. 1988) schlägt Harald Müller-Witt eine grundlegende Neugestaltung hin zu einem medienübergreifenden ökologischen Abgabe-/Steuersystem vor. Für das IÖW hat er wesentliche Gedanken seiner Arbeit unter dem Titel „Progressive Umweltabgaben und ökologische Steuerreform" zusammengefaßt.

Die Kette von Umweltkatastrophen und -skandalen reißt nicht ab. Mit dem Waldsterben, dem Gau von Tschernobyl und dem Ozonloch über der Antarktis, mit dem Treibhauseffekt und seinen Klimakapriolen sowie einer zur Kloake verkommenen Nordsee sind Problemstellungen in das öffentliche Bewußtsein gerückt, die den Durchschnittsbürger von vor zehn Jahren noch überhaupt nicht belasteten. Hinzu kommt die jeweilige Chemiekalie des Monats: Glykol im Wein, Formaldehyd in den Verpackungen, Pestizide und Nitrate im Trinkwasser, Hormone im Kalbfleisch.

Jede einzelne dieser Problemstellungen stellt eine Herausforderung in sich dar, in der Summe

schreien sie geradezu nach einer grundlegenden Weichenstellung in Politik und Wirtschaft.

Angesichts des offenkundig werdenden Versagens der traditionellen Umweltpolitik mit ihren Auflagen, Ge- und Verboten, erlebt der Vorschlag des britischen Ökonomen Arthur C.Pigou aus dem Jahre 1920, von Staatswegen mittels Steuern ökologisch lenkend in den Wirtschaftsablauf einzugreifen, eine beeindruckende Renaissance. Vorläufig allerdings hauptsächlich im $\mathrm{Ge}-$ wand der Abgabe und nur selten als Steuer.

Die Abwasserabgabe, der Kohle- und der Wasserpfennig sind implementierte Instrumente. Abgaben werden darüber hinaus gehandelt für den Bereich Luft auf Schwefel, Stickoxide und Ab-

Fortsetzung S. 10

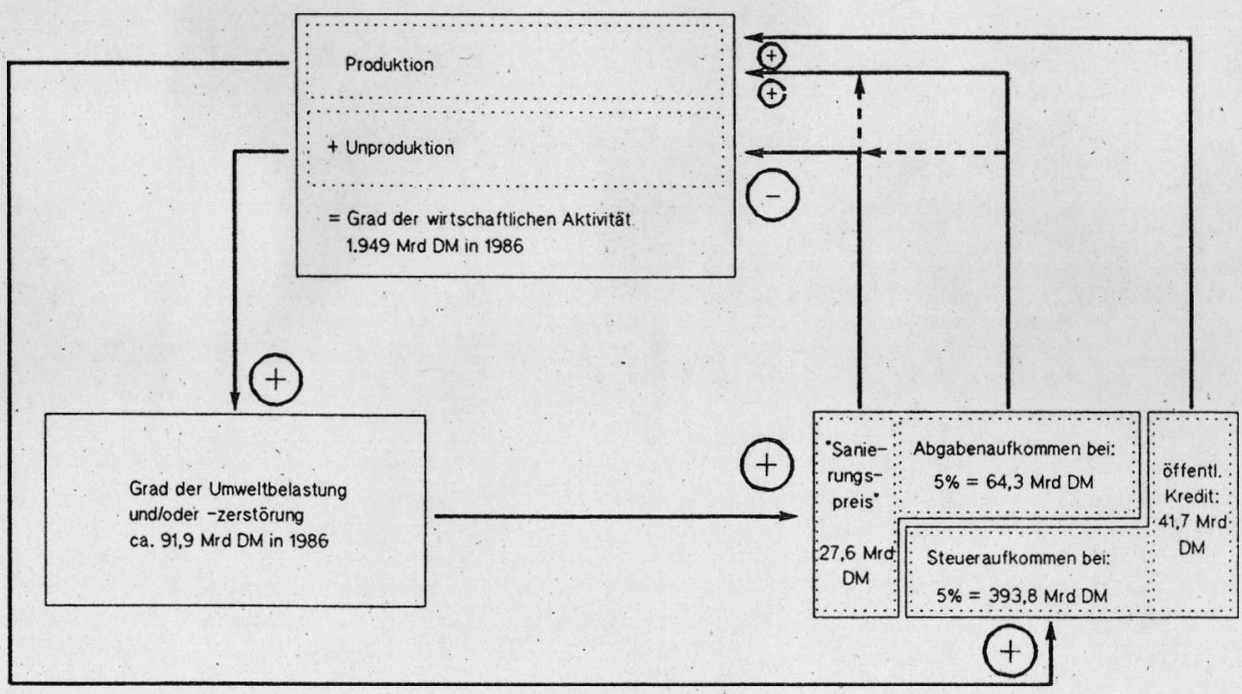

Schaubild: Schematischer Regelkreis Produktionsvolumen / Steueraufkommen / Umweltabgabe
Zur IÖW-Jahrestagung 1988

Ökologischer Konsum

Als ein wesentliches Ergebnis der umweltpolitischen Diskussion kann festgehalten werden, daß die direkten staatlichen Möglichkeiten der Einflußnahme begrenzter und weniger wirkungsvoll sind als anfänglich angenommen.

Von daher wird in zunehmendem Maße die Vielfalt der ökonomischen und politischen Akteure unter dem Gesichtspunkt betrachtet, welche Handlungsmöglichkeiten im einzelnen vorliegen, um zu einer Ökologisierung des Wirtschaftens beizutragen, worin strukturelle und bewußtseinsmäßige Barrieren für solche Handlungsmöglichkeiten bestehen und wie diese abzubauen wären. Die IÖW-Tagung im Dezember 1987 hatte das Thema „Wirtschaftsethik und ökologische Wirtschaftsforschung" (das Buch mit den überarbeiteten Tagungsbeiträgen wird. ca. Febr. 1989 im Haupt-Verlag, Bern, Stuttgart erscheinen). Bei der Bremer VÖW-Jahrestagung im Juni 1988 ging es um „Ökologische Produktionskonzepte" (diese Publikation ist ebenfalls für Anfang 1989 geplant).

Im Rahmen der Frage nach Handlungsmöglichkeiten für ökologisches Wirtschaften war es für uns insofern naheliegend, sich als nächstes dem Problem des Konsumentenverhaltens unter ökologischen Gesichtspunkten zuzuwenden.

Gerade weil hier auf der Ebene der alltäglichen Verhaltensentscheidungen wie der Aktivität von Verbraucherverbänden und -initiativen in jüngster Zeit einiges in Bewegung geraten ist, mangelt es noch an systematischer wissenschaftlicher Reflexion von Konsumentenstrategien, deren Reichweiten und Organisationsbedingungen, den tatsächlichen und möglichen Rückwirkungen auf Produktionsentscheidungen etc. Die geplante Tagung soll diese Reflexion fördern. Ausgehend von den inneren Widersprüchen des alltäglichen Konsumentenverhaltens soll dessen konzeptionellen und strategischen Problemen nachgegangen werden. Es sollen die Erfahrungen ausgewertet werden, die in verschiedenen Handlungsbereichen mit ökolo- 


\section{Fortsetzung v. S. 1}

wärme. Beim Boden auf Stickstoffdünger, Pestizideinsatz und Einwegflaschen. Beim Wasser zusätzlich noch auf die Entnahme von Grundwasser. In jüngster Zeit machte die SPD mit ihrer Energie- und der BUND mit seiner Chemiesteuer von sich reden.

All diesen Vorschlägen gemeinsam ist, daß sie medial isoliert erhoben werden. Insofern unterscheiden sie sich durch nichts von der traditionellen und als fehlerhaft erkannten Umweltpolitik. Was wirklich nottut ist eine medienübergreifende Abgabe/Steuer. Sie gilt es zu fordern und durchzusetzen.

Mit den nachstehenden Gedanken will ich versuchen, hierzu einen Beitrag zu leisten. Dabei werde ich zunächst acht Leitsätze zur Lösung der Umweltproblematik vorstellen, die in der Summe auf die Einführung einer linear-progressiv steigenden Umweltabgabe hinauslaufen. An schließend werde ich Vorschläge zur Finanzierung und zur Verwendung der Abgabe unterbreiten, die sich in der Summe zu einer ökologischen Steuerreform verdichten. Um den Vorschlag plastisch zu machen, werde ich exemplarisch am Beispiel der Luft und mit den ökonomischen Daten von 1986 arbeiten.

1. Es wird ergänzend zu den bestehenden Umweltvorschriften und Gesetzen eine öko-politische $A b$ gabe (oder Steuer) auf jègliche Form von Naturinanspruchnahme erhoben.

2. Diese Abgabe wird jährlich um real 3 Prozent angehoben.

3. Das Aufkommen soll im ersten Jahr der Einführung. 5\% des BSP nicht übersteigen, weitestgehend kostenneutral erhoben und $z u$ annähernd gleichen Teilen aus den Abgaben für die Umweltmedien Boden, Luft und Wasser gespeist werden. 4. Die zu entrichtende Abgabe bemißt sich nach dem physikalischen Maß und der Beschaffenheit des zu reglementierenden Stoffes, gewichtet mit einem spezifischen Hebesatz, in dem sich Umweltgefährdungspotential und Giftigkeit widerspiegeln.

5. Die Abgabe richtet sich unterschiedslos an sämtliche stationären Quellen, gleich ob auf Produzenten-oder Konsumentenseite und an sämtliche mobilen Quellen, soweit man derer habhaft werden kann.

6. Bis zu dem Punkt, an dem die ständig steigende Umweltabgabe die Grenzvermeidungskosten der Unternehmen entsprechend der heutigen umweltgesetzlichen $\mathrm{Ge}$ - und Verbote überspringt, behalten letztere ihre Gültigkeit. Danach werden sie ökonomisch obsolet, juristisch jedoch nicht ungültig.

7. Das Nettoaufkommen aus der Umweltabgabe (der sog. „Sanierungspreis“) wird entsprechend noch zu bestimmender politischer Vorgaben verausgabt. Dabei ist dem Aspekt der Internationalität von Umweltschäden Rechnung zu tragen. Dort, wo eine Subventionsgewährung als Ausweg erscheint, sollten die Mittel gezielt darauf verwandt werden, die "Unproduktion“ einzudämmen.

8. Um für die Subventionsgewährung eine Bezugsgröße und für die. Wirtschaftssubjekte eine Zielvorgabe zu haben, wird die 99\%ige Schad-
Tabelle 1: Preis je Tonne und Umweltschadstoff

\begin{tabular}{|c|c|c|c|c|c|}
\hline Schad- & $\begin{array}{l}\text { Menge } \\
\text { in }\end{array}$ & $\begin{array}{l}\text { Gewich- } \\
\text { tungs - }\end{array}$ & $\begin{array}{l}\text { Gewich- } \\
\text { tungs- }\end{array}$ & $\begin{array}{l}5 X \text { vom BSP } \\
\text { Aufkommen je }\end{array}$ & $\begin{array}{c}\text { 5\% vom BSP } \\
\text { Aboabenpreis in }\end{array}$ \\
\hline stoff & Mio $t$ & $x$ faktor & mass & Schadstoff in OM & DM/Torne Schadst off \\
\hline
\end{tabular}

\begin{tabular}{|c|c|c|c|c|c|c|}
\hline $\mathrm{coO}$ & 800,0 & $x$ & 0,01 & 8,00 & 14.804 .597 .701 & 18,51 \\
\hline co & 7,0 & $x$ & 0,12 & 0,84 & 1.554 .482 .759 & 222,07 \\
\hline 500 & 2,5 & $x$ & 0,75 & 1.88 & 3.479 .080 .460 & 1.391 .63 \\
\hline NOO & 3,0 & $x$ & 0.75 & 2,25 & 4.163 .793 .103 & 1.387 .93 \\
\hline Staub & 0,5 & $x$ & 0,12 & 0,06 & 111.034 .483 & 222,07 \\
\hline - toxisch & 0,1 & $x$ & 0,75 & 0,08 & 148.045 .977 & $1.480,46$ \\
\hline $\mathrm{OCH}$ & 0,5 & $x$ & 0,12 & 0,06 & 111.034 .483 & 222,07 \\
\hline - toxisch & 1,0 & $x$ & 0,75 & 0,75 & 1.387 .931 .034 & $1.387,93$ \\
\hline
\end{tabular}

Summe

$13.92 \quad 25.760 .000 .000$

stoffvermeidung als Ziel proklamiert, welches es binnen 15 Jahren zu realisieren gilt.

Von der Progression und der Höhe des Abgaben(Steuer)Aufkommens im Jahr der Einführung, wird es ganz entscheidend abhängen, wie schnell die Abgabe (Steuer) als Lenkungsinstrument greift. Gegen eine Abgabe, die 5\% des BSP umfaßt, läßt sich nur schwerlich argumentieren. Schließlich entspricht es der herrschenden Rechtsauffassung, daß nach dem Verursacherprinzip niemand Kapital aus einer Schädigung sollte ziehen können, die er der Umwelt und damit der Gemeinschaft zufügt. Bei einem realisierten BSP von 1837,9 Mrd. DM in 1985 hätte das Anfangsaufkommen in 1986 konsequenterweise bei 91,9 Mrd DM liegen müssen.

\section{Wie soll die Umweltabgabe bemessen werden?}

Wegen der Sekundärschädigungen, die die ausgefällten Schadsoffe in den Aufnahmemedien Boden und Wasser anrichten, wählte ich einen Verteilungsschlüssel von 40:30:30 für das Aufkommen aus den Umweltbereichen Luft, Boden und Wasser. Er trägt der Dominanz des Luftpfades Rechnung. Auf die vorgeschlagene 5\% ige Startabgabe bezogen bedeutet das, daß die Umweltbelastung der Luft in 1986 mit 36,8 Mrd DM, die des Bodens mit etwa 27,6 Mrd DM und die des Wassers mit eben der gleichen Summe bei den Verursachern hätte durchschlagen müssen.

Die anfänglichen 36,8 Mrd DM Abgabeaufkommen für die Umweltbelastung der Luft habe ich auf die fünf großen Blöcke Abwärme, Emissionen, Lärm, Strahlung und Gerüche heruntergebrochen. Die Gerüche werden mit 2 Prozent entsprechend 0,74 Mrd DM belastet, die Abwärme mit 3 Prozent, oder 1,1 Mrd DM. Die Emissionen als Hauptgefährdungspotential schlagen mit 70 Prozent, respektive 25,76 Mrd DM zu Buche. Bleiben für Lärm und Strahlung noch 25 übrig. Hiervon sollten 15 Prozent dem Lärm angerechnet werden, das wären 5,52 Mrd DM und 10 Prozent der Strahlung, das wären 3,68 Mrd DM.

In Übereinstimmung mit Pareto konzentrierte ich mich ganz auf die Emissionen, die nach mei- nem Modell 70\% einspielen sollen, und setzte meine Berechnungen auf der Basis von 815 Mio t Schadstoffeintrag fort, als da sind: 800 Mio t $\mathrm{CO}_{2}, 7$ Mio t CO, 2,5 Mio t $\mathrm{SO}_{2}, 3$ Mio t $\mathrm{NO}_{\mathrm{X}}, 0,6$ Mio t Staub und 1,5 Mio t organische Verbindungen. Da Schadstoff nicht gleich Schadstoff ist, macht dies einen Gewichtungsfaktor notwendig, der sich nach dem Umweltgefährdungspotential bemißt.

Im nächsten Schritt galt es das Aufkommen einigermaßen gerecht auf die Emittentengruppen Kraftwerke, Industrie, Haushalte und Kleinverbrauch sowie Verkehr aufzuteilen. Ich griff mir $\mathrm{zu}$ Demonstrationszwecken die öffentliche Stromversorgung und den Kraftfahrzeugverkehr heraus.

1985 betrug die Stromerzeugung mittels fossiler Energieträger $97,1 \mathrm{Mrd} \mathrm{kWh}$ bei Steinkohle, 83,6 Mrd kWh bei Braunkohle, 16,6 Mrd kWh bei Erdgas und 4,4 Mrd kWh bei Heizöl. Aus diesen Zahlen rechnet sich eine durchschnittliche Verteuerung der Kilowattstunde Strom um maximal 6,0 Pf. Nun ist aber nach meinem Modell die Luftabgabe nur für vierzig Prozent des Gesamtaufkommens verantwortlich. Widrigstenfalls käGewässerbelastung nochmals 2,4 Pf hinzu, in der Summe addieren sich die Zusatzkosten auf 8,4 Pf je Kilowattstunde.

Wählt man nun einen durchschnittlichen VierPersonen-Haushalt als Bezugsgröße und setzt dessen Verbrauch mit $3000 \mathrm{kWh} / \mathrm{a}$ an, dann ergibt sich allein bei der. Stromrechnung eine spürbare Mehrbelastung von 252,-DM pro Jahr, entsprechend 21,-DM je Monat. ich auch mit meinem zweiten Beispiel, dem Kraftfahrzeugverkehr. Hier beläuft sich die durch die Emissionsabgabe induzierte Verteuerung des Spritpreises auf maximal $24 \mathrm{Pf}$ je Liter. Im Durchschnitt fährt ein deutscher Autofahrer $12.200 \mathrm{~km}$ pro Jahr und verbraucht dabei $10,7 \mathrm{Li}$ ter auf hundert Kilometer. Aus diesen Zahlen errechnet sich eine durchschnittliche Mehrbelastung je Autofahrer von maximal 313,30 .DM/

Fortsetzung $S .11$ men somit aus der Abgabe für die Boden- und

Auf ähnlich gelagerte Größenordnungen komme 


\section{Fortsetzung v. S. 10}

Jahr; entsprechend 26,11 DM/Monat. - Auch wenn sich hinter diesen Zahlen letztlich nur die tatsächlichen, gesellschaftlichen Kosten des Individualverkehrs verbergen und eine Erhöhung der Benzinpreise um bis zu $40 \mathrm{Pf} / \mathrm{l}$ sich durchaus noch im Rahmen der marktüblichen Preisschwankungsbreite bewegt, derartige Preisschübe bedeuten insbesondere für die Haushalte der unteren und mittleren Einkommensbezieher eine spürbare Mehrbelastung. Dies um so mehr, weil ja parallel zu der emissionsinduzierten Spritpreiserhöhung und den Kostensprüngen beim Strompreis Kostenschübe bei allen anderen Konsumgütern ebenfalls auf der Tagesordnung stehen. Schließlich ist es ja das erklärte Ziel der progressiven Umweltabgaben auf breitester Front und nicht etwa selektiv anzusetzen.

\section{Wie soll die Umweltabgabe finanziert werden?}

Wenn sich aber bereits diese beiden Demonstrationsbeispiele zu einer Netto-Kostenbelastung von 565,30 DM pro Jahr addieren, dann ist leicht abzusehen, daß mein Modell ohne flankierende Maßnahmen rasch die Leistungsfähigkeit eines mittleren Haushalts überschreitet. Nämliches gilt für kleine und mittlere Betriebe. Es kommt also darauf an, durch Steuerminderungen an anderer Stelle, die Einführung der progressiven Umweltabgabe in der Startphase weitestgehend kostenneutral zu gestalten.

Als Neutralisierungsmasse für die Umweltabgabe bieten sich gewisse Steuern geradezu an. Hier schlage ich vor:

die Gewerbesteuern (ca. 32,6 Mrd DM in 1986)

- die Kraftfahrzeugsteuer (ca. 9 Mrd DM in 1986)

- die Mineralölsteuer (ca. 25,2 Mrd DM in 1986) - die „Genußsteuern“ wie z.B. die Kaffeesteuer (1,7 Mrd DM in 1986) und die Zuckersteuer (140 Mio DM in 1986)

ersatzlos zu streichen.

Im Jahr der Einführung der Abgabe strebe ich ein Belastungs-/Entlastungsverhältnis von $1 \mathrm{zu}$ 0,7 an. Dies bedeutet für die bundesdeutschen Produzenten und Konsumenten eine zusätzliche Belastung in Höhe von 27,6 Mrd DM. Der damit einhergehende Realeinkommensverlust der Lohn- und Gewinneinkommensbezieher ist der politisch gewollte, von der Allgemeinheit zu tragende "Sanierungspreis“. Es versteht sich von selbst, daß der dadurch ausgelöste Preisschub auf der Angebotsseite von der Bundesbank durch eine flankierende Geldmengenpolitik abgestützt werden muß. Andernfalls löst sich der Sanierungsimpuls in Inflation auf.

Sofern im ersten Erhebungsjahr keine Einsparerfolge erzielt werden, steigt der „Sanierungspreis“ - wegen der eingebauten 3\% igen Progression im zweiten Laufjahr auf 30,4 Mrd DM. Diese Steigerung darf dann allerdings nicht mehr über den Fortfall anderer Belastungsformen abgepuffert werden, da sonst der angestrebte ökologische Rationalisierungsdruck der Umweltabgabe neutralisiert wird. Gelingen den Wirtschaftssub- jekten Einsparungen im Takt mit der Progression, bleibt das Abgabeaufkommen konstant; wird überproportional zur Progression eingespart, sinkt das Abgabeaufkommen ensprechend (vgl. das Schaubild: Schematischer Regelkreis Produktionsvolumen/Steueraufkommen/Umweltabgabe, S. 1).

Mein Vorschlag korrigiert die vordergründige ökonomische Rationalität des bestehenden Steuersystems, als da sind: die aktive und passive Möglichkeit der Anpassung an die konjunkturellen Erfordernisse (built in flexibility) sowie seine wachstumspolitische Ausrichtung, weil ein $\mathrm{Ko}$ stenblock von $17,43 \%$ am gesamten Steueraufkommen der individuellen Rationalität der Steuerzahler unterworfen wird. Diese nun haben es fortan zu einem Großteil selbst in der Hand, wieviel ihnen vom Einkommen (bzw. Gewinn) abgenommen wird. Je früher, nachhaltiger und stärker sie ihre Präferenzen in Richtung umweltschonende Produkte (respektive Produktion) lenken, desto geringer wird ihr Nettotransfer als Summe aus Steuer- und Abgabenaufkommen ausfallen.

\section{Wie soll das Sondervermögen „Sanierungspreis“"verwendet werden?}

1. Als erstes, wegen der Dringlichkeit, bietet sich ein aus dem „Sanierungspreis“" gespeister, mäßiger Nettokapitalexport in die Länder der 3. Welt an, um der dort galoppierend um sich greifenden Umweltzerstörung gezielt entgegenzusteuern. Hierfür veranschlagen wir 20 Prozent oder 5,2 Mrd DM.

2. Für die Aufstockung der Personal- und Sachausgaben im Umweltschutz habe ich einen Anteil von 15 Prozent vorgesehen. Das wären 4,14 Mrd DM zusätzliche Mittel. Positiver Nebeneffekt dabei: die Aufarbeitung des Vollzugsdefizits mündet in höhere Bußgeldeinnahmen. Die Reform finanziert sich ein Stück weit selbst.

3. Im Bereich der Umweltforschung gibt es erhebliche Lücken. 10 Prozent des Sondervermögens sollten daher der ökologischen Wirkungsforschung, der Erforschung alternativer Energien sowie opto-elektronischer Permanentmeßsysteme im Umweltschutz vorbehalten sein. Der Nettobeschäftigungseffekt dieser zusätzlichen 2,76 Mrd DM liegt rechnerisch bei ca. 24.200 Stellen.

4. Offiziöse Kostenschätzungen für die Sanierung und Überwachung der derzeit lokalisierten Altlasten schwanken in der Größenordnung zwischen 8 und 17 Mrd DM. Aus diesem Grund schlage ich vor, $20 \%$ des Sondervermögens, mithin 5,32 Mrd DM pro Jahr, für die Bildung eines Altlastensanierungsfonds abzuzweigen.

5. Die nächste Anregung verdanke ich dem Philadelphia Fee/Subsidy Modell: eine Subventionierung derjenigen Produzenten, die sich bereits heute in der Lage sehen, das proklamierte Ziel der 99 prozentigen Schadstoffvermeidung einzuhalten. In der Praxis dürfte sich eine Subventionierung ultrasauber arbeitender Industrieanlagen wie ein Turbolader für das Vorantreiben des technischen Fortschritts auswirken. 5 Prozent oder 1,4 Mrd DM des Umweltabgabenaufkommens schlage ich hierfür vor.

6. Nach einer Berechnung des Karl-Bräuer-Insti- tuts des Bundes der Steuerzahler beliefen sich die direkten und indirekten Subventionen an die deutsche Landwirtschaft in 1986 auf rund 31,8 Mrd DM. Von dieser gigantischen Summe erreichte letztendlich nur ein Zehntel die Bauern. Stattdessen schlage ich vor, den Bauern etwas für ihre Arbeit als Landschaftspfleger zu vergüten. Das Karl-Bräuer-Institut hat hierzu ausgerechnet, daß bei einem Übergang auf direkte Einkommenshilfen etwa die Hälfte aller landwirtschaftlichen Betriebe als unterstützungsbedürftig behandelt werden müßten.

Setzt man die durchschnittliche Höhe der direkten Hilfen auf 20.000 DM je Betrieb fest, so wäre dafür ein jährliches Subventionsvolumen von 7,21 Mrd DM erforderlich. Um in etwa diese Dimension realisieren zu können, will ich einen Anteil von 30 Prozent an dem Sondervermögen „Sanierungspreis“ für direkte Einkommenszahlungen reserviert wissen. Je nach Ausgestaltung der Umweltabgabe wäre das zwischen 5,0 und 8,3 Mrd DM pro Jahr.

\section{Welche Vorteile bietet die Abgaben-/Steuerlösung?}

An dieser Stelle gilt es zwischen den administrativen, ökologischen und ökonomischen Vorteilen zu differenzieren. Beginnen möchte ich mit den administrativen.

Der Leser mag sich mein progressives Abgabenmodell wie eine gigantische Bubblelösung vorstellen, die über die gesamte, westdeutsche, später eventuell einmal europäische, Ökonomie gestülpt wird.

Diese „Plastik“glocke ist zunächst einmal so dimensioniert, daß unter ihr alle Emissionen Platz haben, die nach gegenwärtig gültigem Auflagenreglement für die Umweltmedien Boden, Luft und Wasser zulässig sind. An diese Plastikglocke ist - bildlich gesprochen - eine Vakuumpumpe in Form der progressiven Umweltabgaben angeschlossen. Einmal angeworfen, pumpt sie ganz allmählich, aber stetig, die Emissionen heraus. Je größer das Vakuum wird, desto stärker schrumpft die Plastikglocke. um sich im Endstadium (der 99 prozentigen Schadstoffvermeidung) nahezu vollständig an die darunterliegenden Industrien anzuschmiegen. Für Emissionen ist dann kein Raum mehr, der Betrieb würde ökonomisch erdrosselt.

Bis zu diesem Stadium allerdings vergeht einige Zeit. Zeit, die die Betriebe nutzen werden, um im Vorgriff auf die drohenden Abgabensteigerungen ihre Vermeidungsstrategien zu überdenken und Vermeidungsmaßnahmen zu realisieren. Da sich - rationales Verhalten unterstellt - die Betriebe dabei von Grenzvermeidungskosten leiten lassen werden, ist bereits mittelfristig damit zu rechnen, daß sich unter der Glocke das gesamtökonomische Vermeidungsoptimum einstellen wird.

Dabei ist mein Modell weder abhängig von richtig gesetzten Grenzwierten (der Schwäche der traditionellen Auflagenpolitik), noch von der richtig bestimmten Höhe des Abgabensatzes (der Schwäche der bisherigen Abgabenvorschläge)

Fortsetzung $S, 12$ 


\section{Fortsetzung v. S. 11}

und schon gar nicht von der exakt zu bestimmenden Menge der zulässigen Emissionen (der Schwäche der sogenannten Emissionsrechte). Das Modell kann, ohne daß größere Korrekturen gefahren werden müssen, über jede denkbare Variante staatlicher Umweltpolitik gestülpt werden. Einmal implementiert, wirkt die $\mathrm{Ab}$ gabe über die Zeit, einzig aufgrund ihrer eingebauten Progressionsmechanik, wobei der Wirkungsgrad, als Funktion der beiden Variablen Anfangsaufkommen ( $x \%$ vom BSP) und Steigerungsrate ( $\mathrm{y} \%$ pro Jahr) - durch die Vorgaben der Legislative determiniert wird.

Damit erhält der Gesetzgeber einen sehr großen Gestaltungsspielraum, den er je nach politischer Situation, ökologischer Notwendigkeit sowie den ökonomischen Rahmenbedingungen mal enger, mal weiter ziehen kann.

Die Vorteile auf ökologischem Gebiet liegen auf der Hand. Der gegenwärtige Status Quo von Natur und Umwelt wird zum Ausgangspunkt gemacht. Einmal implementiert, wirkt die Abgabe nur noch in Richtung ökologischer Verbesserungen. Sollte es sich in der Umbauphase als notwendig erweisen, den einen oder anderen Schadstoff verschärft zu reglementieren, kann immer wieder auf die Auflagenpolitik zurückgegriffen werden. Das Prinzip der Abgabe, die allmähliche Verdrängung von Auflagen, bleibt davon unberührt.

Ein höchst bedeutsamer ökologischer Vorteil ist, daß es mit der Realisierung eines Modells progressiver Umweltabgaben schlagartig und im höchsten Maße für alle Emittenten unattraktiv wird, von einem Umweltmedium auf ein anderes auszuweichen. Das Ausfindigmachen von Schlupflöchern, bisher noch Aufgabe einer überforderten Administration, die dabei doch nur hinter den betrieblichen Realitäten hinterherhinkt, wäre so in das ökonomische Kalkül, und damit die Eigenverantwortung der Emittenten gestellt. Die derzeit noch völlig falsch gelenkte Energie, die sich nicht selten in kriminellen Aktivitäten auslebt, würde so in strafrechtlich unverfängliche und ökologisch erwünschte Bahnen gelenkt. Das Vermeidbare wird gleich an der Quelle vermieden, das Unvermeidbare in Kaskadenproduktionsketten weitergenutzt, die Abfälle recycliert und was dann noch übrig bleibt, sicher endgelagert.

Nicht nur auf ökologischem, sondern vor allem auch auf ökonomischen Gebiet liegen die Hauptvorzüge eines kombinierten Abgaben-Steuermodells.

Rein rechnerisch nämlich spart das Modell allein auf dem Agrarsektor im Minimum 8,8 Mrd DM pro Jahr, gemessen am derzeitigen Subventionsverfahren aus EG- und anderen staatlichen Mitteln.

Die Entlastung der Konsumentenhaushalte durch marktbestimmte Agrarpreise schlägt, Berechnungen des Karl-Bräuer-Instituts zufolge, mit jährlich 15 Mrd DM zu Buche.

Der bei weitem gewichtigste Vorteil wäre allerdings in der Entlastung auf dem Arbeitsmarkt zu sehen. Belastet man den Ressourceneinsatz, so führt dies zu Preisverschiebungen die den Ein- satz von menschlicher Arbeitskraft rentabler und den von Rohstoffen unrentabler macht.

Überträgt man die Berechnungen von Frank Springmann (Steuerreform zum Abbau von Arbeitslosigkeit und Umweltbelastung. Ein Szenario. WZB IIUG dp 86-11) auf mein Modell linear-progressiv steigender Umweltabgaben, welches 91,9 Mrd DM einspielen soll, so bedeutet dies eine glatte Halbierung, eventuell sogar Drittelung der bestehenden Arbeitslosigkeit

Die durch den Beschäftigungsschub bewirkten Steuermehreinnahmen des Staates einerseits, sowie seine Einsparungen beim Wohngeld und der Sozialhilfe andererseits, saldieren sich mit Leichtigkeit zu einem zweistelligen Milliardenbetrag. Damit nicht genug: Gesetzt den Fall, es gelänge durch die Abgabe tatsächlich den säkulären Trend zur Substitution von Arbeit durch Kapital und Rohstoffe (Energie) in sein Gegenteil zu verkehren und damit zugleich den Druck auf die Umweltressourcen zu mildern, dann ergeben sich materielle Verbesserungen (grob vereinfacht) als:

- Einsparungen der öffentlichen Körperschaften bei den Ausgaben zur Beseitigung von Umweltschäden

- Verringerung der Defizite im öffentlichen Personennahverkehr und bei der Bundesbahn

- Verringerung der Kosten für Sanierungs-, Konservierungs- und sonstige Maßnahmen zur Verlängerung der Lebensdauer von Kulturgütern, öffentlichen Gebäuden und Bauwerken (bspw. der Infrastruktur)

- Werterhaltung der staatlichen Forste und Ländereien

- Einsparungen bei den Kosten für das Gesundheitswesen.

Aus dem bislang Dargelegten leite ich für meinen Vorschlag progressiver Umweltabgaben folgende Erkenntnisse ab:

1. das Modell ist finanzierbar. Dies gilt für den Teil, der durch Wegfall anderer Steuerarten belastungsneutral erhoben wird.

2. das Modell ist tragbar. Dies gilt für den Teil, der als „Sanierungspreis" die Steuerzahler zusätzlich belastet.

Die Gewißheit für die zweite Erkenntnis leite ich aus einer vergleichenden Betrachtung der Steuerquoten ab. 1985 lag sie bei 23,9 Prozent vom Bruttosozialprodukt. Legt man ein Umweltabgabenaufkommen für 1986 mit fünf Prozent vom BSP des Jahres 1985 zu Grunde, dann wäre die "Steuerquote“ 1986 auf 24,9\% - gemessen als Steuerbelastung plus Abgabenaufkommen (incl. Sanierungspreis) - gestiegen. Sie hätte damit lediglich die Höchstmarke egalisiert, die wir 1977 in der Bundesrepublik verzeichneten. Damals allerdings ohne den Beitrag zur Umweltsanierung. Angesichts der in diesem Artikel zusammengetragenen massiven Vorteile bleibt zu fragen, weshalb die ökologische Steuerreform nicht schon längst auf der Tagesordnung aller progressiven Kräfte steht. Es gilt jetzt, die neuen Mehrheiten zur Durchsetzung zu organisieren.

Harald Müller-Witt, Ratingen

\section{Termine}

15.Sept. SPD-Berlin: Forum Fabrik 2000 - Zukunft der Arbeit in der Industriegesellschaft

16.-18.Sept. Friedrich-Ebert-Stiftung Bergneustadt: Arbeit und Umwelt in NRW-Zwischenbilanz und politische Perspektiven bei der ökonomischen und ökologischen Erneuerung unseres Landes

23.-25.Sept. Ev. Akademie Arnoldshain: Eingriffe des Menschen in die Stoffkreisläufe der Natur I: Energiegewinnung

22.-24.9. Deutscher Naturschutzring (in Berlin anläß1. IWF/Weltbank-Tagung): Umweltzerstörung und Weltbank. Umwelt und Schuldenkrise in der Dritten Welt

25.-30.Sept. Gesellschaft für Ökologie: 18. Jahrestagung in Essen

28.Sept. Ministerium für Arbeit, Gesundheit und Soziales NRW, Messe Köln: Fachtagung Mensch und Technik im Alltag

30.Sept. Institut für ökologische Zukunftsperspektiven Barsinghausen: Die Umweltverträglichkeitsprüfung in Kommunen

3.-6.Okt. Deutsches Institut für Urbanistik Berlin: Wirtschaftsförderung und Umweltvorsorge. Ökologische Orientierung eines kommunalen Aufgabenbereichs?

5.-6.Okt. AGP, DQCG, IMT, RKW; Bad Dürkheim: 7. deutscher Quality Circle Kongreß

6./7.Okt. Ev. Akademie Bad Boll: Recyclingpapier im Spannungsfeld von Ökonomie, Ökologie, Maschinentauglichkeit und ästhetischen Ansprüchen

7.-9.Okt. Ev. Akademie Hofgeismar: Hat der Fortschritt eine Zukunft ? Sozialdemokratische Programmatik jenseits der Industriemoderne

6.-9.Okt. Ev.Akademikerschaft i.D., Ruhruni Bochum: Symposium: Wirtschaften im Jahr 2000 - mit welchen Konzepten?

13.Okt. Arbeitskreis Umweltschutz im VDI, Bremen: Umweltrecht und Haftung - Wie sind die Tendenzen?

4.-6.Nov. Ev. Akademie Bad Boll: Ökologische Steuerreform

7.-10. Nov. CC Hamburg: Climate and Development. World Congress

10./11.Nov. Arbeitsgemeinschaft für wirtschaftl. Verwaltung, Eschborn: Tagung in Boppard: Organisation des Umweltschutzes - Für eine bessere Zusammenarbeit und Wirtschaftlichkeit

10.-12.Nov. Gh Kassel, Fb Stadt-/Landschaftsplanung: Theorie und Praxis eigenständiger Regionalentwicklung im ländlichen Raum

11./12.Nov. Ev. Akademie Tutzing: Ökologische Steuerungsmöglichkeiten des Staates

14.Nov. future-forum 1988 in Düsseldorf: Von der Öko-Bilanz zum Öko-Controlling. Möglichkeiten und Grenzen umweltorientierter Unternehmenspolitik

14.-16.Nov. Ev. Akademie Loccum: Umdenken Umlenken: Zur ökologischen Verantwortung des Staates

15.Nov. Gottlieb Duttweiler Institut Rüschlikon/ Zürich: Kulturelle Modernisierung in Unternehmung und Gesellschaft

18.-20.Nov. Ev. Akademie Baden, Bad Herrenalb: Die Arbeit der Zukunft

14.-16.Dez. Ev. Akademie Loccum: Wandel des Arbeitsbegriffes der 80er Jahre - von der Realität eingeholt? 
(c) 20I0 Authors; licensee IÖW and oekom verlag. This is an article distributed under the terms of the Creative Commons Attribution Non-Commercial No Derivates License (http://creativecommons.org/licenses/by-nc-nd/3.o/), which permits unrestricted use, distribution, and reproduction in any medium, provided the original work is properly cited. 